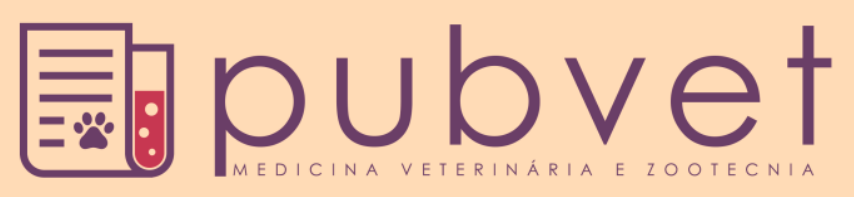

HTTP://DX.DOI.ORG/10.22256/PUBVET.V11N11.1119-1122

\title{
Eletroforese urinária do cão séptico: Revisão
}

\author{
Breno Curty Barbosa ${ }^{1 *}$, Luiz Eduardo de Souza Tassini ${ }^{1}$, Dayse Helena Lages Silva ${ }^{2}$, \\ Daniela Bastos de Souza Karam Rosa ${ }^{2}$, Fellipe Ferreira Nogueira ${ }^{3}$, Paulo Ricardo de \\ Oliveira Paes ${ }^{4}$, Fabíola Oliveira Paes Leme ${ }^{4}$ \\ ${ }^{1}$ Médico Veterinário doutorando em Ciência Animal da Universidade Federal de Minas Gerais UFMG, Departamento de \\ Clínica e Cirurgia Veterinárias. Belo Horizonte-MG, Brasil.E-mail: brenocurty@hotmail.com \\ ${ }^{2}$ Médico Veterinário mestrando em Ciência Animal da Universidade Federal de Minas Gerais UFMG. Departamento de \\ Clínica e Cirurgia Veterinárias. Belo Horizonte - MG, Brasil. \\ ${ }^{3}$ Médico Veterinário autônomo. Belo Horizonte - MG, Brasil \\ ${ }^{4}$ Médico Veterinário docente da Universidade Federal de Minas Gerais UFMG. Departamento de Clínica e Cirurgia \\ Veterinárias. Belo Horizonte - MG, Brasil.
}

RESUMO. Os quadros sépticos são constantes na rotina do setor de terapia intensiva, sendo a injúria renal aguda a principal disfunção orgânica observada nesses pacientes, contribuindo para a alta taxa de mortalidade. O diagnóstico precoce de lesão renal nesses quadros é de fundamental importância e contribui de forma positiva ao prognóstico. Os métodos diagnósticos da lesão renal, entretanto ainda são restritos. A eletroforese permite separar proteínas de baixo peso molecular na urina, podendo representar um marcador precoce de lesão associado com um direcionamento da terapêutica adotada.

Palavras Chave: cão, eletroforese urinária, sepse

\section{Electrophoresis urinary of septic dogs: Review}

\begin{abstract}
The septic conditions are constant in the routine of the intensive care sector, with acute renal injury being the main organic dysfunction observed in these patients, contributing to the high mortality rate. Early diagnosis of renal damage in these settings is of fundamental importance and contributes positively to the prognosis. The diagnostic methods of renal injury, however, are still restricted. Electrophoresis allows the separation of low molecular weight proteins in the urine and may represent an early marker of injury associated with a targeting of the therapy adopted.
\end{abstract}

Keyworlds: Dog, urinary electrophoresis, sepsis

\section{Electroforesis urinaria del perro séptico: Revisión}

RESUMEN. Los cuadros sépticos son constantes en la rutina del sector de terapia intensiva, siendo la injuria renal aguda la principal disfunción orgánica observada en esos pacientes, contribuyendo para la alta tasa de mortalidad. El diagnóstico precoz de lesión renal en estos cuadros es de fundamental importancia y contribuye de forma positiva al pronóstico. Los métodos diagnósticos de la lesión renal, sin embargo todavía son restringidos. La electroforesis permite separar proteínas de bajo peso molecular en la orina, pudiendo representar un marcador precoz de lesión asociado con un direccionamiento de la terapéutica adoptada.

Palabras clave: Perro, electroforesis urinaria, sepsis 


\section{Introdução}

Os quadros sépticos são responsáveis pelo maior atendimento de rotina clínica do setor de emergência e terapia intensiva em Medicina Veterinária. Em estudo realizado por Barbosa et al. (2016) os quadros de sepse ocuparam o primeiro lugar dos casos atendidos nesse setor no período de um ano, sendo responsáveis por $17 \%$ da rotina do Hospital Veterinário da Escola de Veterinária da UFMG. Alguns autores consideram que a taxa de óbito de pacientes com sepse esteja entre 30 e 50\% (Schaefer et al., 2011), o que ressalta a importância de se caracterizar o paciente séptico para que melhores abordagens terapêuticas possam ser empregadas.

A injúria renal aguda (IRA) é a disfunção orgânica mais comum nos pacientes críticos decorrente de sepse e ocorre por diversos mecanismos patofisiológicos (hipoperfusão, injuria de isquemia e reperfusão glomerular, estresse oxidativo, entre outros). Sabe-se, porém, que a injúria precede a perda de função e, dentre as principais funções renais está a função excretora. Esta função, normalmente, é avaliada através dos exames de ureia e creatinina séricas em situações de suspeita de injúria renal, entretanto, apresentam alteração de seus valores apenas quando há progressão de uma injúria a ponto de comprometer de 66 a $75 \%$ dos néfrons de ambos os rins do paciente, dessa forma tem sido considerados marcadores tardios (Bellomo et al., 2012). A possibilidade de realizar um diagnóstico precoce, ou seja, identificar a injúria antes de haver perda de função renal, pode contribuir para um melhor prognóstico e maior sobrevida dos pacientes em quadros de sepse e IRA. Dentre os métodos de diagnóstico precoce, podemos citar a avaliação e classificação de proteínas urinárias, pela eletroforese (Schaefer et al., 2011, Alobaidi et al., 2015).

\section{Sepse e suas definições}

Como definição os quadros sépticos podem ser classificados em síndrome da resposta inflamatória sistêmica (SIRS), síndrome sepse, sepse grave e choque séptico respectivamente e, segundo Rabelo (2013), se deve a intensidade em que se desenvolve o desequilíbrio entre a produção e a liberação das citocinas pró e antiinflamatórias. De acordo com Theobaldo (2012) o paciente em SIRS deve cursar com ao menos duas variáveis descritas na Tabela 1. Já para ser enquadrado em síndrome sepse, deve apresentar
SIRS em associação há infecção presumida ou comprovada oriunda por vírus, fungos ou bactérias. Nos quadros de sepse grave se encaixam os pacientes que cursam, além dos sinais clínicos da síndrome sepse, ao menos uma disfunção orgânica descrita no Quadro 1, com a ressalva da reversão dos quadros hipotensivos apenas utilizando as manobras de restituição volêmica. Caso necessite usar vasopressores e inotrópicos para normalização dos quadros pressóricos ele já se encaixa em choque séptico.

Tabela 1. Valores de variáveis fisiológicas em cão com quadro de SIRS.

\begin{tabular}{lll}
\hline Variável & Cão \\
\hline Temperatura retal & $<38,1^{\circ} \mathrm{C}$ ou $>39,2^{\circ} \mathrm{C}$ & \\
Frequência cardíaca & $>120 \mathrm{bpm}$ \\
Frequência respiratória & $>20 \mathrm{mpm}$ \\
Leucócitos totais; bastonetes & $<6 \quad \mathrm{x} 10^{3} / \mathrm{mm}^{3} \quad$ ou $\quad>$ \\
& $16 \times 10^{3} / \mathrm{mm}^{3} ;>3 \%$ & \\
\hline
\end{tabular}

Fonte: Hauptman et al. (1997).

Quadro 1. Disfunções orgânicas associadas ao quadro de sepse grave em cão.

Hipotensão: PAM < 80mmHg ou PAS < 120mmHg

- Hipotensão grave: queda abrupta de mais de $40 \mathrm{mmHg}$ na PAS ou PAM $<65 \mathrm{mmHg}$

- Oligúria $(<2 \mathrm{ml} / \mathrm{kg} / \mathrm{h})$ ou Creatinina $>2 \mathrm{mg} / \mathrm{Dl}$

- Hiperbilirrubinemia: $>0,5 \mathrm{mg} / \mathrm{dL}$

- Consciência alterada: Glasgow pediátrico modificado $<17$ ou AVDN < A

- Disfunção respiratória

- Trombocitopenia: $<50.000 / \mathrm{mm}^{3}$ ou queda de mais de $50 \%$ em 12 horas

- Aumento de TP, TTPA/D-dímero ou queda de fibrinogênio

- Íleo paralítico

- $\quad$ Albumina $<2,5 \mathrm{~g} / \mathrm{Dl}$

PAM: pressão arterial média; PAS: pressão arterial sistólica; AVDN: alerta, responsivo a comando verbal, responsivo à dor, não responsivo; TP: tempo de protrombina; TTPA: tempo de tromboplastina ativada. Adaptado de $\underline{\text { Rabelo }}$ $\underline{(2012)}$

\section{Disfunção renal na sepse}

Disfunção mais comum no paciente séptico, a IRA, que possui como classificação uma redução abrupta da função renal, acarretando em distúrbios sistêmicos de grande importância piorando o prognóstico do paciente séptico (Birchard and Sherding, 2008, Alobaidi et al., 2015). Os sinais clínicos resultam da incapacidade dos rins em excretar resíduos metabólicos e controlar adequadamente o equilíbrio hidroeletrolítico e acidobásico (Xavier et al., 2008). 
A hiperfosfatemia e o aumento de ureia e creatinina são os analítos bioquímicos mais observados (Birchard and Sherding, 2008). Entretanto, têm sido considerados tardios, inespecíficos e pouco sensíveis (Coca et al., 2008). Os biomarcadores precoces são avaliados individualmente ou em painéis (plasmáticos ou urinários) para identificar e monitorar a injúria inicial e a duração da IRA. Devido às diferentes expressões dos marcadores, pode ser possível determinar o tipo de IRA de acordo com sua etiologia. Dentre as formas mais promissoras de monitoramento, estão os painéis plasmáticos (NGAL e Cistatina C) e os painéis urinários (NGAL, IL-18 e KIM-1) (Majumdar, 2010). Como exemplo, pode-se citar a Lipocalina Associada à Gelatinase Neutrofílica (NGAL) como conclui o estudo de Bagshaw et al. (2010) envolvendo 83 humanos adultos críticos com IRA, destes 43 eram sépticos. Os pacientes em sepse apresentaram valores maiores da NGAL tanto plasmática quanto urinária, e nos pacientes não sépticos o seu pico foi um preditor de progressão.

A avaliação clínica da disfunção renal precoce representa um desafio na prática clínica veterinária, pois o diagnóstico realizado na rotina clínica expõe uma lesão já tardia (Giori et al., 2011). Em contrapartida à perda de função está a renoproteção. A renoproteção pode ser entendida como um conjunto de terapias, que visa prevenir a instalação de uma injúria ou monitorar e evitar a progressão de uma injúria instalada. Como exemplos de terapias renoprotetoras, podemos citar o uso de drogas inibidoras da enzima conversora de angiotensina (IECA), uso de ácidos graxos do tipo ômega-3, controle do fósforo dietético, monitoramento da pressão arterial e métodos de diagnóstico precoce (Ruggenenti et al., 2003). O prognóstico de cães com IRA está relacionado de forma direta com a precocidade do diagnóstico baseado nos resultados dos exames laboratoriais associados aos exames clínicos, juntamente com a resposta ao tratamento da injuria, sendo o diagnóstico da lesão renal antes de torna-se IRA o objetivo principal na terapêutica do paciente crítico, pois assim é possível garantir um melhor prognóstico e uma melhor recuperação em menor tempo. (Giori et al., 2011, Bragato, 2013).

\section{Eletroforese urinária}

Em destaque, a eletroforese vem mostrando cada vez mais o seu potencial para diagnostico na detecção de proteínas séricas e urinárias provenientes de alterações inflamatórias, infecciosas ou imunológicas ou tubulares e glomerulares, respectivamente (Cintra et al., 2015). Com essa técnica é possível avaliar o local da lesão no parênquima renal de acordo com o peso da proteína encontrada (Oliveira et al., 2015). A técnica de eletroforese, utilizando o sodium dodecyl sulphate-polyacrylamide gel electrophoresis (SDS-PAGE) é uma técnica não invasiva, marca um número maior de proteínas urinária do que a eletroforese simples e consegue inferir a origem da proteína. As de origem glomerular apresentam pesos moleculares maiores que $80 \mathrm{kDa}$,como as imunoglobulinas $\mathrm{G}$ (que não demonstra seletividade glomerular), ou entre $60 \mathrm{e}$ $80 \mathrm{kDa}$, como a albumina. As de origem tubular são difíceis de caracterizar, pois apresentam baixo peso molecular (menor que $60 \mathrm{kDa}$ ) como a proteína de ligação ao retinol (RBP), que passa pelo glomérulo renal, porém é reabsorvida no túbulo proximal. Reconhecer a presença dessa proteína e sua estratificação pode direcionar o tratamento e o prognóstico, se tornando uma ferramenta muito útil para os pacientes sépticos (Giori et al., 2011, Schaefer et al., 2011).

Estudo dirigido por Schaefer et al. (2011), no qual foram usadas urinas de 39 cães diagnosticado com SIRS além de 15 cães como grupo controle, apresentou resultados promissores, pois os cães em SIRS apresentaram 11 bandas no gel e os saudáveis apenas 3, o que demonstra uma maior perda proteica em pacientes com sepse instalada. Dos animais em SIRS 58\% das bandas eram de baixo peso molecular comparada com o grupo controle (45\%) $42 \%$ das bandas apresentavam peso molecular médio a baixo no grupo SIRS comparado a 55\% observados no grupo controle. Embora os resultados tenham se mostrado promissores os autores concluíram que novos estudos são necessários para se verificar se a magnitude da proteinúria é preditivo de gravidade nos pacientes em sepse (Schaefer et al., 2011).

\section{Considerações Finais}

Há uma necessidade de se implantar na rotina clínica veterinária novos métodos de diagnóstico precoce da injuria renal aguda nos pacientes sépticos, haja vista o elevado índice de mortalidade associado com a baixa realização da hemodiálise e transplante renal. A eletroforese urinária é uma técnica promissora, que vem se destacando pela sensibilidade em quantificar a presença urinária de proteínas de baixo peso molecular e que, futuramente, poderá ser uma 
opção de marcador e direcionador da terapia a ser adotada em paciente sépticos.

\section{Referências Bibliográficas}

Alobaidi, R., Basu, R. K., Goldstein, S. L. \& Bagshaw, S. M. 2015. Sepsis-associated acute kidney injury. Seminars in nephrology. Elsevier.

Bagshaw, S. M., Bennett, M., Haase, M., HaaseFielitz, A., Egi, M., Morimatsu, H., D’amico, G., Goldsmith, D., Devarajan, P. \& Bellomo, R. 2010. Plasma and urine neutrophil gelatinase-associated lipocalin in septic versus non-septic acute kidney injury in critical illness. Intensive Care Medicine, 36, 452-461.

Barbosa, B.C.; Alves, F.S.; Santos, W.G.; Beier, S.L.; Paes, P.R.O.; Freitas, P.M.C. 2016 Levantamento dos casos atendidos no setor de emergência e terapia intensiva da escola de veterinária da universidade federal de minas gerais de dezembro de 2014-dezembro de 2015. In: Semana de Educação Continuada em Medicina Veterinária da UFES, 13, 2016, Alegre. Anais da XIII SECOMV. Alegre 2016. p. 19. Resumo.

Bellomo, R., Kellum, J. A. \& Ronco, C. 2012. Acute kidney injury. The Lancet, 380, 756766.

Birchard, S. J. \& Sherding, R. G. 2008. Manual Saunders: clínica de pequenos animais, São Paulo.

Bragato, N. 2013. Fisiologia renal e insuficiência renal aguda em pequenos animais: Causas e consequências. Escola de Veterinária $e$ Zootecnia. Universidade Federal de Góias, Goiânia.

Cintra, C. A. A., Souza, G. C., Ribeiro, G. F. D., Silva, P. C., Heitor, T. F., Coelho, S. B., Coutin, C. C., Tedesco, A. T., Nascimento, M. R. \& Magalhães, L. F. 2015. Importância da técnica de eletroforese na avaliação de alterações túbulo-glomerulares em cães. Investigação, 14, 51-54.

Coca, S. G., Yalavarthy, R., Concato, J. \& Parikh, C. R. 2008. Biomarkers for the diagnosis and risk stratification of acute kidney injury: a systematic review. Kidney International, 73, 1008-1016.
Giori, L., Tricomi, F. M., Zatelli, A., Roura, X. \& Paltrinieri, S. 2011. High-resolution gel electrophoresis and sodium dodecyl sulphateagarose gel electrophoresis on urine samples for qualitative analysis of proteinuria in dogs. Journal of Veterinary Diagnostic Investigation, 23, 682-690.

Hauptman, J. G., Walshaw, R. \& Olivier, N. B. 1997. Evaluation of the sensitivity and specificity of diagnostic criteria for sepsis in dogs. Veterinary Surgery, 26, 393-397.

Majumdar, A. 2010. Sepsis-induced acute kidney injury. Indian Journal of Critical Care Medicine, 14, 14-21.

Oliveira, E., Trentin, T. C., Camargo, F., Pinto, Y. D. P. \& Martins, D. B. 2015. Eletroforese: Conceitos e aplicações. Enciclopédia Biosfera Centro Científico Conhecer, 22, 1129-1149.

Rabelo, R. 2012. Emergências em pequenos animais: Condutas clínicas e cirúrgicas no paciente grave. Elsevier Brasil, Rio de Janeiro.

Ruggenenti, P., Perna, A. \& Remuzzi, G. 2003. Retarding progression of chronic renal disease: the neglected issue of residual proteinuria. Kidney International, 63, 2254-2261.

Schaefer, H., Kohn, B., Schweigert, F. J. \& Raila, J. 2011. Quantitative and qualitative urine protein excretion in dogs with severe inflammatory response syndrome. Journal of Veterinary Internal Medicine, 25, 1292-1297.

Theobaldo, M. C. 2012. Efeitos da solução salina hipertônica na resposta inflamatória na sepse. Universidade de São Paulo, São Paulo.

Xavier, A., Puzzi, M., B., Polizer, K. \& 2008., L. F. 2008. Insuficiência renal aguda. Revista Científica Eletrônica de Medicina Veterinária da Faculdade de Medicina Veterinária $e$ Zootecnia de Garça, 10, 1-4.

\section{Article History:}

Received 2 August 2017

Accepted 25 August 2017

Available on line 1 September 2017

License information: This is an open-access article distributed under the terms of the Creative Commons Attribution License 4.0, which permits unrestricted use, distribution, and reproduction in any medium, provided the original work is properly cited. 